\title{
Warum hängen alle so an diesem Unsinn?
}

\author{
Ökonomen im Gesundheitssystem führen zu Kostenexplosionen
}

Immer wieder versuchen Ökonomen das Gesundheitssystem in Zahlen abzubilden, was natürlich erkenntnistheoretisch nicht geht. Abgesehen davon, dass es in den Arbeitszeiten und Gehaltszahlungen enorme Unterschiede zwischen privat und öffentlich gibt, hat die Medizin nun evidence-based nachgewiesen, dass ökonomische Interventionen zur Kostenexplosion führen, weil es, wie McKinsey (unverdächtig) nachweist, erst zu Einsparungen kommt, wenn Ärzte in den Führungspositionen stehen und mitentscheiden.

Im Hauptverband der Sozialversicherungsträger und vielen Kassen sitzen vor allem Ökonomen, die keine Ahnung von Medizin haben (woher auch, sie haben ja noch nie Patienten behandelt und können sich das nicht vorstellen). Diese propagieren, dass z. B. Vorsorgeuntersuchungen Einsparungen bringen. Seit Jahren ist bekannt, dass Vorsorgeuntersuchungen mit wenigen Ausnahmen vor allem zusätzliche Kosten verursachen und fast nichts zur Gesundheit der Bevölkerung beitragen. Warum sollen eine Anamnese und ein Status, durchgeführt an einer 23-jähri-

\footnotetext{
Univ.-Prof. Dr. Kaspar Sertl,

Systemischer Organisationsberater,

Gystemischer Organisation

Primarius, Pianist, Wien
}

gen, ein längeres und gesünderes Leben ergeben. Das ergibt a priori keinen Sinn.

Aber es gibt noch ganz andere Hinweise: In den USA wurden die Polizzenkosten für ambulante Arztbesuche für ältere Patienten erhöht, um Kosten zu sparen. Auf so eine Idee können nur Ökonomen kommen. Die Patienten gingen tatsächlich weniger oft zum Arzt und wurden dann kränker ins Spital eingeliefert, mussten dort länger liegen und die Gesamtkosten explodierten.

In North Carolina wurden Millionen Dollar für Qualitätsmanagement ausgegeben (zumeist nur eingeführt, um Kosten zu sparen), an der Qualität änderte sich nach sieben Jahren nichts, aber das Geld war weg. Mittels Disease Management wollte man die Kosten senken, indem man Patienten intensiver ambulant betreute. Dies ist wirklich weltweit gescheitert. So kam es also zu keiner Senkung der Spitalsaufenthalte und zu keiner Senkung der Zahl der Notfallsambulanzbesuche - und auch zu keinen Einsparungen.

Sodann erfanden Ökonomen in England „pay for performance“. Außer, dass die Ärzte nach Einführung dieses Systems weniger gut für die Patienten erreichbar und Millionen Pfund weg waren, hat auch dieses System nichts gebracht - auch qualitativ nicht.

Ebenso wurden alle „total quality systeme“ (ISO, EFQM, Balance Score Card etc.) dazu erfunden, die Kosten in den Griff zu bekommen. Seit Jahren zeigt sich, auch von der Österreichischen Wirtschaftskammer bestätigt, dass es zu keiner Verbesserung der Performance von Spitälern kommt, aber dass diese Systeme unglaublich teuer sind und das Personal vor allem an der Arbeit am Patienten behindern.

Die Liste an Beispielen ließe sich beliebig fortsetzen. Es ist ganz klar und gut dokumentiert, dass auch alle ELGA-Systeme für die Patienten gar nichts bringen (sondern insgesamt potenziell gefährlich sind), strategisch nichts bringen und zu einer Verteuerung führen. Warum hängen alle so an diesem Unsinn? Was bringt den Befürwortern das Befürworten? Der EDV-Industrie Millionen oder Milliarden? Vermeintliche Macht? Vermeintliche Kontrolle über Ärzte? Es ist und bleibt unklar, dafür ist es aber zu teuer.

Also: Beenden wir diese teuren unsinnigen Qualitätssysteme! Wir würden unglaublich viel Geld sparen und könnten unsere Qualität wieder erhöhen, indem wir uns wieder mit Patienten statt mit Computern auseinandersetzen.

\section{Korrespondenz:}

Univ.-Prof. Dr. Kaspar Sertl

E-Mail: kaspar.sert|@wienkav.at

\section{IMPRESSUM}

Herausgeber und Verleger: Springer-Verlag GmbH, Professional Media, Sachsenplatz 4-6, P.O.Box 89, 1201 Wien, Austria, Tel.: $01 / 33024$ 15, Fax: $01 / 33024$ 26-260, Internet: www.springer.at, www.SpringerMedizin at; Geschäftsführung: Dr. Alois Sillaber. Peter Hendriks, Harm van Maanen; Leitung Professional Media: Mag. Margarete Zupan; Redaktion: Verantwortlicher Redakteur: Dr. Verena Kienast; Mitarbeiter dieser Ausgabe: Dr. Renate Höhl, Gabriele Popernitsch; Redaktionssekretariat: Susanna Hinterberger; Portalmanagement SpringerMedizin.at: Andrea Niemann; Produktion: Mag. (FH) Dorothea Woliński; Anzeigen: Dipl. Tzt. Elise Haidenthaller; Es gilt die Anzeigenpreisliste 2012; Erscheinungsweise: $6 x$ jährlich; Abonnement: Michaela Bolli; Wiener klinisches Magazin ist eine Beilage zur Wiener klinischen Wochenschrift (WKW); Bezugspreis pro Jahr: EUR 47,- zuzüglich MwSt. und Versandkosten; Verlagsort: Wien; Herstellungsort: Wien; Erscheinungsort: Wien; Verlagspostamt: 1210 Wien P.b.b.; ISSN Print: 1869-1757; ISSN Electronic: 1613-7817; Band 14, Heft 3/2012; Layout: Melanie Marschall; Design: Wojtek Grzymala; Druck: Holzhausen Druck GmbH, 1140 Wien-Auhof, Holzhausenplatz 1.

Alle namentlich gekennzeichneten Beiträge spiegeln nicht unbedingt die Meinung der Redaktion wider. Diese Beiträge fallen somit in den persönlichen Verantwortungsbereich des Verfassers. Die Redaktion übernimmt keine Haftung für unaufgefordert eingesandte Manuskripte. Mit "Sonderbericht" oder "Advertorial" gekennzeichnete Seiten sind entgeltliche Einschaltungen nach $\$ 26$ Mediengesetz. Allgemeiner Teil/Rechtliche Hinweise für Autoren: Die Autorin/der Autor erklärt, dass ihr/sein Manuskript in dieser Form bislang nicht anderweitig veröffentlicht oder zur Veröffentlichung eingereicht wurde. Die Autorin/der Autor überträgt mit der Übergabe des fertigen Manuskripts und der Veröffentlichung in der Fachzeitschrift die notwendigen Nutzungsrechte zur Vervielfältigung und Verbreitung an den Verlag, insbesondere das Recht der Nutzung zu gewerblichen Zwecken durch Druck, Nachdruck, Verbreitung in elektronischer Form oder andere Verfahren und Medien durch Springer Science + Business Media. Die Autorin/der Autor holt, falls notwendig, die Nutzungsrechte an Texten und Bildern Dritter vor Übergabe des fertigen Manuskripts ein, eventuelle Ansprüche Dritter sind somit geklärt. Hinweise zur Verwertung: Die Zeitschrift sowie alle in ihr enthaltenen einzelnen Beiträge und Abbildungen sind urheberrechtlich geschützt. Jede Verwertung, auch auszugsweise, die nicht ausdrücklich vom Urhebre in dieser Zeitschrift berechtigt auch ohne besondere Kennzeichnung nicht zu der Annahme, dass solche Namen im Sinne der Warenzeichen- und Markenschutz-Gesetzgebung als frei zu betrachten wären und daher von jedermann benutzt werden dürften. Angaben über Dosierungsanweisungen und Applikationsformen sind anhand anderer Literaturstellen oder der Packungsbeilage auf ihre Richtigkeit zu überprüfen. Der Verlag übernimmt hierfür keine Gewähr.

Eigentümer und Copyright-Inhaber: () 2012 Springer-Verlag/Wien. Springer ist Teil der Fachverlagsgruppe Springer Science+Business Media; Beilage zur Wiener klinischen Wochenschrift 17-18/2012 